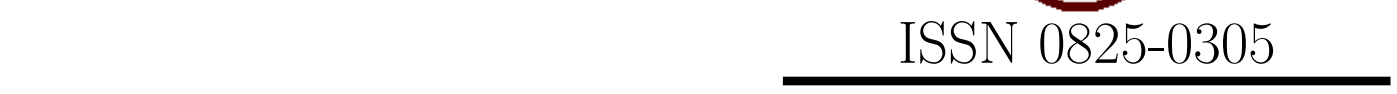

\title{
On error probability exponents of many hypotheses optimal testing illustrations
}

\author{
Leader Navaei \\ Payame Noor University, 19395-4697, Tehran, I.R. of Iran \\ Received 28 January 2011; Accepted 23 September 2011 \\ Copyright (c) 2011, Journal Afrika Statistika. All rights reserved
}

\begin{abstract}
In this paper we study a model of hypotheses testing consisting of with to simple homogeneous stationary Markov chains ith finite number of states such that having different distributions from four possible transmission probabilities.For solving this problem we apply the method of type and large deviation techniques (LTD). The case of two objects having different distributions from to given probability distribution as examined by Ahlswedeh and Haroutunian.

Résumé. Dans cet article nous étudions un modèle de tests d'hypothèses composé de deux chaines de Markov stationnaires homogènes et simples avec un nombre fini d'états ayant différentes distributions parmi quatre probabilités de transition possibles. Pour résoudre ce problème, nous appliquons la méthode des types et des techniques de grandes deviations. Le cas de deux objets ayant différentes distributions issues d'une distribution de probabilité donnée, a été examiné par Ahlswedeh et Haroutunian.
\end{abstract}

Key words: Markov chains; Error probabilities; Different distributions; Transition probabilities; Reliabilities.

AMS 2010 Mathematics Subject Classification : 62P30; 62M02.

\section{Introduction}

Applications of information-theoretical methods in mathematical statistics are reflected in the monographs by Kullback [10], Csiszár and Körner [4], Blahut [2], Csiszár and Shields [5], Zeitouni and Gutman [14]. In the book of Csiszár and Shields [5] different asymptotic aspects of two hypotheses testing for independent identically distributed observations are considered via theory of large deviations. Similar problems for Markov dependence of experiments were investigated by Natarajan [13], Haroutunian [7], [8], Haroutunian and Navaei [9] and others.

Ahlswede and Haroutunian in [1] formulated an ensemble of problems on multiple hypotheses testing for many objects and on identification of hypotheses under reliability requirement. 
The problem of many $(L>2)$ hypotheses testing on distributions of independent observations is studied in [13], [11] via large deviations techniques.

In this paper we investigate a model with two simple homogeneous stationary Markov chains with finite number of states such that having different distributions from four possible transition probabilities. In Section 2 we introduce the concept of Markov chain and the method of type [3] and in Section 3, we apply the result Section 2 for hypotheses testing.

\section{Preliminaries}

Let $\mathbf{y}=\left(y_{0}, y_{1}, y_{2}, \ldots, y_{N}\right), y_{n} \in \mathcal{Y}=\{1,2, \ldots, I\}, \mathbf{y} \in \mathcal{Y}^{N+1}, N=0,1,2, \ldots$, be a vectors of observations of a simple homogeneous stationary Markov chain with finite number $I$ of states. The $l=\overline{1, L}$ hypotheses concern the irreducible matrices of the transition probabilities

$$
P_{l}=\left\{P_{l}(j \mid i), i=\overline{1, I}, j=\overline{1, I}\right\}, \quad l=\overline{1, L} .
$$

The stationarity of the chain provides existence for each $l=\overline{1, L}$ of the unique stationary distribution $Q_{l}=\left\{Q_{l}(i), i=\overline{1, I}\right\}$, such that

$$
\sum_{i} Q_{l}(i) P_{l}(j \mid i)=Q_{l}(j), \quad \sum_{i} Q_{l}(i)=1, \quad i=\overline{1, I}, \quad j=\overline{1, I} .
$$

We define the joint distributions

$$
Q_{l} \circ P_{l}=\left\{Q_{l}(i) P_{l}(j \mid i), i=\overline{1, I}, j=\overline{1, I}\right\}, l=\overline{1, L} .
$$

Let us denote $D\left(Q \circ P \| Q_{l} \circ P_{l}\right)$ Kullback-Leibler divergence

of the distribution

$$
\begin{aligned}
D\left(Q \circ P \| Q_{l} \circ P_{l}\right) & =\sum_{i, j} Q(i) P(j \mid i)\left[\log Q(i) P(j \mid i)-\log Q_{l}(i) P_{l}(j \mid i)\right] \\
& =D\left(Q \| Q_{l}\right)+D\left(Q \circ P \| Q \circ P_{l}\right),
\end{aligned}
$$

$$
Q \circ P=\{Q(i) P(j \mid i), i=\overline{1, I}, j=\overline{1, I}\},
$$

with respect to distribution $Q_{l} \circ P_{l}$ where

$$
D\left(Q \| Q_{l}\right)=\sum_{i} Q(i)\left[\log Q(i)-\log Q_{l}(i)\right], l=\overline{1, L} .
$$

Let us name the second order type of vector $\mathbf{y}$ the square matrix of $I^{2}$ relative frequencies $\left\{N(i, j) N^{-1}, i=\overline{1, I}, j=\overline{1, I}\right\}$ of the simultaneous appearance in $\mathbf{y}$ of the states $i$ and $j$ on the pairs of neighbor places. It is clear that $\sum_{i j} N(i, j)=N$. Denote by $\mathcal{T}_{Q \circ P}^{N}$ the set of vectors from $\mathcal{Y}^{N+1}$ which have the second order type such that for some joint $\mathrm{PD} Q \circ P$

$$
N(i, j)=N Q(i) P(j \mid i), \quad i=\overline{1, I}, \quad j=\overline{1, I} .
$$

The set of all joint $\mathrm{PD} Q \circ P$ on $\mathcal{Y}$ is denoted by $\mathcal{Q} \circ \mathcal{P}(\mathcal{Y})$ and the set of all possible the second order types for joint PD $Q \circ P$ is denoted by $\mathcal{Q} \circ \mathcal{P}^{N}(\mathcal{Y})$. Note that if vector $\mathbf{y} \in \mathcal{T}_{Q \circ P}^{N}$, then

Journal home page: www.jafristat.net 


$$
\sum_{j} N(i, j)=N Q(i), i=\overline{1, I}, \quad \sum_{i} N(i, j)=N Q^{\prime}(j), j=\overline{1, I},
$$

for somewhat different from $\mathrm{PD} Q^{\prime}$, but in accordance with the definition of $N(i, j)$ we have

$$
\left|N Q(i)-N Q^{\prime}(i)\right| \leq 1, i=\overline{1, I},
$$

and then in the limit, when $N \rightarrow \infty$, the distribution $Q$ coincides with $Q^{\prime}$ and may be taken as stationary for conditional PD $P$ :

$$
\sum_{i} Q(i) P(j \mid i)=Q(j), j \in \mathcal{Y}
$$

The probability of vector $\mathbf{y} \in \mathcal{Y}^{N+1}$ of the Markov chain with transition probabilities $P_{l}$ and stationary distribution $Q_{l}$, is the following

$$
\begin{gathered}
Q_{l} \circ P_{l}^{N}(\mathbf{y}) \triangleq Q_{l}\left(y_{0}\right) \prod_{n=1}^{N} P_{l}\left(y_{n} \mid y_{n-1}\right), l=\overline{1, I}, \\
Q_{l} \circ P_{l}^{N}(\mathcal{A}) \triangleq \sum_{\mathbf{y} \in \mathcal{A}} Q_{l} \circ P_{l}^{N}(\mathbf{y}), \mathcal{A} \subset \mathcal{Y}^{N+1} .
\end{gathered}
$$

Note also that if $Q \circ P$ is absolutely continuous relative to $Q_{l} \circ P_{l}$, then from [3],[7] we have

$$
Q_{l} \circ P_{l}^{N}\left(\mathcal{T}_{Q \circ P}^{N}\right)=\exp \left\{-N\left(D\left(Q \circ P \| Q \circ P_{l}\right)\right)+o(1)\right\},
$$

where

$$
\begin{gathered}
o(1)=\max \left(\max _{i}\left|N^{-1} \log Q_{l}(i)\right|: Q_{l}(i)>0\right), \\
\left(\max _{i}\left|N^{-1} \log Q_{l}(i)\right|: Q_{l}(i)>0\right) \rightarrow 0, \quad \text { when } N \rightarrow \infty .
\end{gathered}
$$

and also according [5],[8] this is not difficult to verify taking into account that the number $\left|\mathcal{T}_{Q \circ P}^{N}\right|$ of vectors in $\mathcal{T}_{Q \circ P}^{N}$ is equal to

$$
\exp \left\{-N\left(\sum_{i, j} Q(i) P(j \mid i) \log P(j \mid i)\right)+o(1)\right\} .
$$

In the next section we use the results of this section for the case of $L=12$ Hypotheses LAO testing.

\section{Problem Statement and Formulation of Results}

Let $Y_{1}$ and $Y_{2}$ be random variables (RV) taking values in the same finite set $\mathcal{Y}$ with one of $L=4$ PDs.

Let $\left(\mathbf{y}_{\mathbf{1}}, \mathbf{y}_{\mathbf{2}}\right)=\left(\left(y_{0}^{1}, y_{0}^{2}\right), \ldots,\left(y_{n}^{1}, y_{n}^{2}\right), \ldots,\left(y_{N}^{1}, y_{N}^{2}\right)\right), y^{i} \in \mathcal{Y}, \quad i=1,2, \quad n=\overline{0, N}$, be a sequence of results of $N+1$ independent observations of a simple homogeneses stationary Markov chain with finite number $I$ of states. The goal of the statistician is to define which 
joint of distributions corresponds to observed sample $\left(\mathbf{y}_{\mathbf{1}}, \mathbf{y}_{\mathbf{2}}\right)$, which we denote by $\phi_{N}$. For this model the vector $\left(Y_{1}, Y_{2}\right)$ can have one of six joint probability distributions $Q_{l_{1}, l_{2}}^{\prime} \circ$ $P_{l_{1}, l_{2}}^{\prime}\left(\mathbf{y}_{1}, \mathbf{y}_{\mathbf{2}}\right), l_{1} \neq l_{2}, l_{1}, l_{2}=\overline{1,4}$ where

$$
Q_{l_{1}, l_{2}}^{\prime} \circ P_{l_{1}, l_{2}}^{\prime}\left(\mathbf{y}_{1}, \mathbf{y}_{2}\right)=Q_{l_{1}}^{\prime} \circ P_{l_{1}}^{\prime}\left(\mathbf{y}_{1}\right) Q_{l_{2}}^{\prime} \circ P_{l_{2}}^{\prime}\left(\mathbf{y}_{\mathbf{2}}\right)
$$

We can take $\left(Y_{1}, Y_{2}\right)=X, \mathcal{Y} \times \mathcal{Y}=\mathcal{X}$ and $\mathbf{x}=\left(x_{0}, x_{1}, x_{2}, \ldots, x_{N}\right), x_{n} \in \mathcal{X}, \mathbf{x} \in \mathcal{X}^{N+1}$, where $x_{n}=\left(y_{n}^{1}, y_{n}^{2}\right), n=\overline{0, N}$, then we will have six new hypotheses for one object.

$$
\begin{array}{ll}
Q_{1,2}^{\prime} \circ P_{1,2}^{\prime}\left(\mathbf{y}_{\mathbf{1}}, \mathbf{y}_{\mathbf{2}}\right)=Q_{1} \circ P_{1}(\mathbf{x}), & Q_{2,1}^{\prime} \circ P_{2,1}^{\prime}\left(\mathbf{y}_{\mathbf{1}}, \mathbf{y}_{\mathbf{2}}\right)=Q_{4} \circ P_{4}(\mathbf{x}), \\
Q_{1,3}^{\prime} \circ P_{1,3}^{\prime}\left(\mathbf{y}_{\mathbf{1}}, \mathbf{y}_{\mathbf{2}}\right)=Q_{2} \circ P_{2}(\mathbf{x}), & Q_{2,3}^{\prime} \circ P_{2,3}^{\prime}\left(\mathbf{y}_{\mathbf{1}}, \mathbf{y}_{\mathbf{2}}\right)=Q_{5} \circ P_{5}(\mathbf{x}), \\
Q_{1,4}^{\prime} \circ P_{1,4}^{\prime}\left(\mathbf{y}_{\mathbf{1}}, \mathbf{y}_{\mathbf{2}}\right)=Q_{3} \circ P_{3}(\mathbf{x}), & Q_{2,4}^{\prime} \circ P_{2,4}^{\prime}\left(\mathbf{y}_{\mathbf{1}}, \mathbf{y}_{\mathbf{2}}\right)=Q_{6} \circ P_{6}(\mathbf{x}), \\
& \\
Q_{3,1}^{\prime} \circ P_{3,1}^{\prime}\left(\mathbf{y}_{\mathbf{1}}, \mathbf{y}_{\mathbf{2}}\right)=Q_{7} \circ P_{7}(\mathbf{x}), & Q_{4,1}^{\prime} \circ P_{4,1}^{\prime}\left(\mathbf{y}_{\mathbf{1}}, \mathbf{y}_{\mathbf{2}}\right)=Q_{10} \circ P_{10}(\mathbf{x}), \\
Q_{3,2}^{\prime} \circ P_{3,2}^{\prime}\left(\mathbf{y}_{\mathbf{1}}, \mathbf{y}_{\mathbf{2}}\right)=Q_{8} \circ P_{8}(\mathbf{x}), & Q_{4,2}^{\prime} \circ P_{4,2}^{\prime}\left(\mathbf{y}_{\mathbf{1}}, \mathbf{y}_{\mathbf{2}}\right)=Q_{11} \circ P_{11}(\mathbf{x}), \\
Q_{3,4}^{\prime} \circ P_{3,4}^{\prime}\left(\mathbf{y}_{\mathbf{1}}, \mathbf{y}_{\mathbf{2}}\right)=Q_{9} \circ P_{9}(\mathbf{x}), & Q_{4,3}^{\prime} \circ P_{4,3}^{\prime}\left(\mathbf{y}_{1}, \mathbf{y}_{\mathbf{2}}\right)=Q_{12} \circ P_{12}(\mathbf{x}),
\end{array}
$$

and thus we have brought the original problem to the identification problem for one object of observation of Markov chain with finite number of states with $L=12$ hypotheses.

Now, according non-randomized test $\phi_{N}(\mathbf{x})$ accepts one of the hypotheses $H_{l}, l=\overline{1,12}$ on the basis of the trajectory $\mathbf{x}=\left(x_{0}, x_{1}, \ldots, x_{N}\right)$ of the $N+1$ observations. Let us denote $\alpha_{l \mid m}^{(N)}\left(\phi_{N}\right)$ the probability to accept the hypothesis $H_{l}$ under the condition that $H_{m}, m \neq l$, is true. For $l=m$ we denote $\alpha_{m \mid m}^{(N)}\left(\phi_{N}\right)$ the probability to reject the hypothesis $H_{m}$. It is clear that

$$
\alpha_{m \mid m}^{(N)}\left(\phi_{N}\right)=\sum_{l \neq m} \alpha_{l \mid m}^{(N)}\left(\phi_{N}\right), m=\overline{1,12}
$$

This probability is called the error probability of the $m$-th kind of the test $\phi_{N}$. The quadratic matrix of 144 error probabilities $\left\{\alpha_{l \mid m}^{(N)}(\phi), m=\overline{1,12}, l=\overline{1,12}\right\}$ sometimes is called the power of the tests. To every trajectory $\mathbf{x}$ the test $\phi_{N}$ puts in correspondence one from 6 hypotheses. So the space $\mathcal{X}^{N+1}$ will be divided into 12 parts, $\mathcal{G}_{l}^{N}=\left\{\mathbf{x}, \phi_{N}(\mathbf{x})=l\right\}, l=$ $\overline{1,12}$, and $\alpha_{l \mid m}^{N}\left(\phi_{N}\right)=Q_{m} \circ P_{m}\left(\mathcal{G}_{l}^{N}\right), \quad m, l=\overline{1,12}$.

We study the matrix of "reliabilities",

$$
E_{l \mid m}(\phi)=\lim _{N \rightarrow \infty}-\frac{1}{N} \log \alpha_{l \mid m}\left(\phi_{N}\right), m, l=\overline{1,12}
$$

Note that from definitions (1) and (2) it follows that

$$
E_{m \mid m}=\min _{l \neq m} E_{l \mid m}
$$




$$
\mathbf{E}(\phi)=\left[\begin{array}{ccccc}
E_{1 \mid 1} & \ldots & E_{1 \mid m} & \ldots & E_{1 \mid 12} \\
\vdots & & \vdots & & \vdots \\
E_{l \mid 1} & \ldots & E_{l \mid m} & \ldots & E_{l \mid 12} \\
\vdots & & \vdots & & \vdots \\
E_{12 \mid 1} & \ldots & E_{12 \mid m} & \ldots & E_{12 \mid 12}
\end{array}\right]
$$

Definition 1. The test sequence $\Phi^{*}=\left(\phi_{1}, \phi_{2}, \ldots\right)$ is called LAO if for given family of positive numbers $E_{1 \mid 1}, E_{2 \mid 2}, \ldots, E_{11 \mid 11}$, the reliability matrix contains in the diagonal these numbers and the remained 133 its components take the maximal possible values.

Let $P=\{P(j \mid i)\}$ be a irreducible matrix of transition probabilities of some stationary Markov chain with the same set $\mathcal{X}$ of states, and $Q=\{Q(i), i=\overline{1, I}\}$ be the corresponding stationary PD.

For given family of positive numbers $E_{1 \mid 1}, E_{2 \mid 2}, \ldots, E_{11 \mid 11}$, let us define the decision rule $\phi^{*}$ by the sets

$$
\begin{gathered}
\mathcal{R}_{l} \triangleq\left\{Q \circ P: D\left(Q \circ P \| Q \circ P_{l}\right) \leq E_{l \mid l}, \quad D\left(Q \| Q_{l}\right)<\infty\right\}, l=\overline{1,11}, \\
\mathcal{R}_{6} \triangleq\left\{Q \circ P: D\left(Q \circ P \| Q \circ P_{l}\right)>E_{l \mid l}, l=\overline{1,11}\right\}, \\
\mathcal{R}_{l}^{N} \triangleq \mathcal{R}_{l} \cap \mathcal{Q} \circ \mathcal{P}^{N}(\mathcal{X}), \quad l=\overline{1,12} .
\end{gathered}
$$

and introduce the functions:

$$
\begin{gathered}
E_{l \mid l}^{*}\left(E_{l \mid l}\right) \triangleq E_{l \mid l}, l=\overline{1,11}, \\
E_{l \mid m}^{*}\left(E_{l \mid l}\right)=\inf _{Q \circ P \in \mathcal{R}_{l}} D\left(Q \circ P \| Q \circ P_{m}\right), m=\overline{1,12}, l \neq m, l=\overline{1,11} \\
E_{12 \mid m}^{*}\left(E_{1 \mid 1}, \ldots, E_{11 \mid 11}\right) \triangleq \inf _{Q \circ P \in \mathcal{R}_{12}} D\left(Q \circ P \| Q \circ P_{m}\right), m=\overline{1,11},
\end{gathered}
$$

and

$$
E_{12 \mid 12}^{*}\left(E_{1 \mid 1}, \ldots, E_{11 \mid 11}\right) \triangleq \min _{l=1,11} E_{l \mid 12}^{*} .
$$

We cite the statement of the general case of large deviation result for types by Natarajan [13].

Theorem 1. : Let $\mathcal{X}=\{1,2, \ldots, I\}$ be a discrete topological space of finite set of the states of the stationary Markov chain possessing an irreducible transition matrix $P$ and $(\mathcal{X}, \mathcal{A})$ be a measurable space such that $\mathcal{A}$ be a nonempty and open subset or convex subset of joint distributions $Q \circ P$ and $Q_{m}$ is stationary distribution for $P_{m}$, then for the type $Q \circ P(\mathbf{x})$ of a vector $\mathbf{x}$ from $Q_{m} \circ P_{m}$ on $\mathcal{X}$ :

$$
\lim _{N \rightarrow \infty}-\frac{1}{N} \log Q_{m} \circ P_{m}^{N}\{\mathbf{x}: Q \circ P(\mathbf{x}) \in \mathcal{A}\}=\inf _{Q \circ P \in \mathcal{A}} D\left(Q \circ P \| Q \circ P_{m}\right) .
$$


In this section we use the following lemma.

Lemma 1. If elements $E_{m \mid l}\left(\varphi^{i}\right), \quad m, l=\overline{1,12}, i=1,2$, are strictly positive, then the following equalities hold for $\Phi=\left(\varphi^{1}, \varphi^{2}\right)$ :

$$
\begin{aligned}
& E_{m_{1}, m_{2} \mid l_{1}, l_{2}}(\Phi)=E_{m_{1} \mid l_{1}}\left(\varphi^{1}\right)+E_{m_{2} \mid l_{2}}\left(\varphi^{2}\right), \quad \text { if } \quad m_{1} \neq l_{1}, \quad m_{2} \neq l_{2}, \\
& E_{m_{1}, m_{2} \mid l_{1}, l_{2}}(\Phi)=E_{m_{i} \mid l_{i}}\left(\varphi^{i}\right), \quad \text { if } \quad m_{3-i}=l_{3-i} \quad m_{i} \neq l_{i}, \quad i=1,2 .
\end{aligned}
$$

Proof : From the independence of the objects we can write:

$$
\begin{gathered}
\alpha_{m_{1}, m_{2} \mid l_{1}, l_{2}}^{N}\left(\Phi_{N}\right)=\alpha_{m_{1} \mid l_{1}}\left(\varphi^{1}\right) \alpha_{m_{2} \mid l_{2}}\left(\varphi^{2}\right), \quad \text { if } \quad m_{1} \neq l_{1}, \quad m_{2} \neq l_{2}, \\
\alpha_{m_{1}, m_{2} \mid l_{1}, l_{2}}^{N}\left(\Phi_{N}\right)=\alpha_{m_{i} \mid l_{i}}\left(\varphi_{N}^{i}\right)\left[1-\alpha_{m_{3}-i \mid l_{3}-i}\right]\left(\varphi_{N}^{3-i}\right), \quad \text { if } \quad m_{3}-i \neq l_{3}-i, \quad m_{i} \neq l_{i}
\end{gathered}
$$

According to the definitions (1) and (2) we obtain (a) and (b) from equalities (c) and (d).

Notice that using Lemma 1 , for joint probability distributions $D\left(Q_{l_{1}, l_{2}}^{\prime} \circ P_{l_{1}, l_{2}}^{\prime} \| Q_{m_{1}, m_{2}}^{\prime}\right)$ and definition of $\alpha_{l \mid m}^{N}\left(\phi_{N}\right)=Q_{m} \circ P_{m}\left(\mathcal{G}_{l}^{N}\right), \quad m, l=\overline{1,12}$, it is clear that: When $m_{i}, l_{i}=\overline{1,4}, i=$ $1,2, m_{1} \neq m_{2}, l_{1} \neq l_{2}$, we have

$$
\begin{gathered}
D\left(Q_{l_{1}, l_{2}}^{\prime} \circ P_{l_{1}, l_{2}}^{\prime} \| Q_{m_{1}, m_{2}}^{\prime} \circ P_{m_{1}, m_{2}}^{\prime}\right)= \\
D\left(Q_{l_{1}}^{\prime(1)} \circ P_{l_{1}}^{\prime(1)} \| Q_{m_{1}}^{\prime(1)} \circ P_{m_{1}}^{\prime(1)}\right)+D\left(Q_{l_{2}}^{\prime(2)} \circ P_{l_{2}}^{\prime(2)} \| Q_{m_{2}}^{\prime(2)} \circ P_{m_{2}}^{\prime(2)}\right),
\end{gathered}
$$

and for $m_{i} \neq l_{i}, m_{3-i}=l_{3-i}, i=1,2$,

$$
D\left(Q_{l_{1}, l_{2}}^{\prime} \circ P_{l_{1}, l_{2}}^{\prime} \| Q_{m_{1}, m_{2}}^{\prime} \circ P_{m_{1}, m_{2}}^{\prime}\right)=D\left(Q_{l_{i}}^{\prime(i)} \circ P_{l_{i}}^{\prime(i)} \| Q_{m_{i}}^{\prime(i)} \circ P_{m_{i}}^{(i)}\right) .
$$

For example

$$
D\left(Q_{1,2}^{\prime} \circ P_{1,2}^{\prime} \| Q_{4,2}^{\prime} \circ P_{4,2}^{\prime}\right)=D\left(Q_{1}^{\prime(1)} \circ P_{1}^{\prime(1)} \| Q_{4}^{\prime(1)} \circ P_{4}^{\prime(1)}\right)
$$

Now we formulate the theorem from [9], which we prove by application of Theorem 1.

Theorem 2. Let $\mathcal{X}$ be a fixed finite set, and $P_{1}, \cdots, P_{12}$ be a family of distinct distributions of a Markov chain. Consider the following conditions for positive finite numbers $E_{1 \mid 1}, \cdots, E_{11 \mid 11}$ :

$$
\begin{gathered}
0<E_{1 \mid 1}<\min \left[D\left(Q_{m} \circ P_{m} \| Q_{m} \circ P_{1}\right), m=\overline{2,12}\right], \\
0<E_{l \mid l}<\min \left[\min E_{l \mid m}^{*}\left(E_{m \mid m}\right)_{m=\overline{1, l-1}}, \min D\left(Q_{m} \circ P_{m} \| Q_{m} \circ P_{l}\right)_{m=\overline{l+1,12}}\right], \\
l=\overline{2,11 .}
\end{gathered}
$$

Two following statements hold: 
a). if conditions (6) are verified, then here exists a LAO sequence of tests $\phi^{*}$, the reliability matrix of which $E^{*}=\left\{E_{l \mid m}^{*}\left(\phi^{*}\right)\right\}$ is defined in (5), and all elements of it are positive,

b). even if one of conditions (6) is violated, then the reliability matrix of an arbitrary test having in diagonal numbers $E_{1 \mid 1}, \cdots, E_{11 \mid 11}$ necessarily has an element equal to zero (the corresponding error probability does not tend exponentially to zero).

Proof: First we remark that $D\left(Q \circ P_{l} \| Q \circ P_{m}\right)>0$, for $l \neq m$, because all measures $P_{l}, l=\overline{1,12}$, are distinct. Let us prove the statement a) of the Theorem 2 about the existence of the sequence corresponding to a given $E_{1 \mid 1}, \cdots, E_{11 \mid 11}$ satisfying condition (6). Consider the following sequence of tests $\phi^{*}$ given by the sets

$$
\mathcal{B}_{l}^{N}=\bigcup_{Q \circ P \in \mathcal{R}_{l}^{N}} \mathcal{T}_{Q \circ P}^{N}(\mathbf{x}), \quad l=\overline{1,12} .
$$

Notice that on account of condition (6) and the continuity of divergence $D$ for $N$ large enough the sets $\mathcal{R}_{l}^{N}, l=\overline{1,12}$ from (4) are not empty. The sets $\mathcal{B}_{l}^{N}, l=\overline{1,12}$, satisfy conditions :

$$
\mathcal{B}_{l}^{N} \bigcap \mathcal{B}_{m}^{N}=\emptyset, l \neq m, \quad \bigcup_{l=1}^{12} \mathcal{B}_{l}^{N}=\mathcal{X}^{N} .
$$

Now let us show that, exponent $E_{l \mid m}\left(\phi^{*}\right)$ for sequence of tests $\phi^{*}$ defined in $(7)$ is equal to $E_{l \mid m}^{*}$. We know from (4) that $\mathcal{R}_{l}, l=\overline{1,11}$, are convex subset and $\mathcal{R}_{12}$ is open subset of the decision rule of $\phi^{*}$, therefore $\mathcal{R}_{l}, l=\overline{1,12}$, satisfy in condition of Theorem 1 . With relations (4), (5), by Theorem 1 we have

$$
\lim _{N \rightarrow \infty}-\frac{1}{N} \log \alpha_{l \mid m}^{N}\left(\phi^{*}\right)=\lim _{N \rightarrow \infty}-\frac{1}{N} \log Q_{m} \circ P_{m}^{N}\left(\mathcal{R}_{l}\right)=\inf _{Q \circ P \in \mathcal{R}_{l}} D\left(Q \circ P \| Q \circ P_{m}\right) .
$$

Now using (2) and (8) we can write

$$
E_{l \mid m}\left(\phi^{*}\right)=\inf _{Q \circ P \in \mathcal{R}_{l}} D\left(Q \circ P \| Q \circ P_{m}\right) \quad m, l=\overline{1,12} .
$$

Using (8), (4) and (5) we can see that all $E_{l \mid m}^{*}$ are strictly positive. The proof of part (a) will be finished if one demonstrates that the sequence of the tests $\phi^{*}$ is LAO, that is for given finite $E_{1 \mid 1}, \cdots, E_{11 \mid 11}$ for any other sequence of tests $\phi^{* *}$

$$
E_{l \mid m}^{*}\left(\phi^{* *}\right) \leq E_{l \mid m}^{*}\left(\phi^{*}\right), \quad m, l=\overline{1,12} .
$$

Let us consider another sequence of tests $\phi^{* *}$, which is defined by the sets $\mathcal{G}_{1}^{N}, \cdots, \mathcal{G}_{12}^{N}$ such that

$$
E_{l \mid m}^{*}\left(\phi^{* *}\right) \geq E_{l \mid m}^{*}\left(\phi^{*}\right), \quad m, l=\overline{1,12} .
$$

This condition is equivalent to the inequality

$$
\alpha_{l \mid m}^{*}\left(\phi^{* *}\right) \leq \alpha_{l \mid m}^{*}\left(\phi^{*}\right)
$$


We examine the sets $\mathcal{G}_{l}^{N} \cap \mathcal{B}_{l}^{N}, l=\overline{1,11}$. This intersection can not be empty, because in that case

$$
\begin{gathered}
\alpha_{l \mid l}^{(N)}\left(\phi^{* *}\right)=Q_{l} \circ P_{l}^{N}\left(\overline{\mathcal{G}_{l}^{N}}\right) \geq Q_{l} \circ P_{l}^{N}\left(\mathcal{B}_{l}^{N}\right) \geq \\
\max _{Q} \max _{Q \circ D\left(Q \circ P \| Q_{l} \circ P_{l}\right) \leq E_{l \mid l}} Q_{l} \circ P_{l}^{(N)}\left(\mathcal{T}_{Q \circ P}^{N}(\mathbf{x})\right) \geq \exp \left\{-N\left(E_{l \mid l}+o(1)\right)\right\}
\end{gathered}
$$

Let us show that $\mathcal{G}_{l}^{N} \cap \mathcal{B}_{m}^{N}=\emptyset, l=\overline{1,11}$. If there exists $Q \circ P$ such that $D\left(Q \circ P \| Q_{l} \circ P_{l}\right) \leq E_{l \mid l}$ and $\mathcal{T}_{Q \circ P}^{N}(\mathbf{x}) \in \mathcal{G}_{l}^{N}$, then

$$
\alpha_{l \mid m}^{(N)}\left(\phi^{* *}\right)=Q_{m} \circ P_{m}^{N}\left(\overline{\mathcal{G}_{l}^{N}}\right)>Q_{m} \circ P_{m}^{N}\left(\mathcal{T}_{Q \circ P}^{N}(\mathbf{x})\right) \geq \exp \left\{-N\left(E_{m \mid m}+o(1)\right)\right\}
$$

When $0 \neq \mathcal{G}_{l}^{N} \cap \mathcal{T}_{Q \circ P}^{N}(\mathbf{x}) \neq \mathcal{T}_{Q \circ P}^{N}(\mathbf{x})$, we also obtain that

$$
\alpha_{l \mid m}^{(N)}\left(\phi^{* *}\right)=Q_{m} \circ P_{m}^{N}\left(\mathcal{G}_{l}^{N}\right)>Q_{m} \circ P_{m}^{N}\left(\mathcal{G}_{l}^{N} \bigcap \mathcal{T}_{Q \circ P}^{N}(\mathbf{x})\right) \geq \exp \left\{-N\left(E_{m \mid m}+o(1)\right)\right\}
$$

Thus it follows if

a). $l<m$ from (6)we obtain that $E_{l \mid m}\left(\phi^{* *}\right) \leq E_{m \mid m}<E_{l \mid m}^{*}\left(\phi^{*}\right)$.

b). $l>m$ then $E_{l \mid m}\left(\phi^{* *}\right) \leq E_{m \mid m}<E_{l \mid m}^{*}\left(\phi^{*}\right)$, which contradicts our assumption. Hence we obtain that $\mathcal{G}_{l}^{N} \cap \mathcal{B}_{l}^{N}=\mathcal{B}_{l}^{N}, l=\overline{1,11}$. The following intersection $\mathcal{G}_{12}^{N} \cap \mathcal{B}_{12}^{N}=\mathcal{B}_{12}^{N}$ is empty too, because otherwise

$$
\alpha_{12 \mid l}^{*}\left(\phi^{* *}\right) \geq \alpha_{12 \mid l}^{*}\left(\phi^{*}\right)
$$

which contradicts to (10), in this case $\mathcal{G}_{l}^{N}=\mathcal{B}_{l}^{N}, l=\overline{1,12}$.

According the previous explaining the statement of part b) of theorem is evident, since the violation of one of the conditions (8) reduces to the equality to zero of a least one of the elements $E_{l \mid m}^{*}$ defined in (5).

\section{References}

[1] Ahlswede, R. F. and Haroutunian, E. A., 2006. Testing of hypotheses and identification. Lecture Notes in Computer Science, vol. 4123. "General Theory of Information Transfer and Combinations", Springer, pp. 462-478.

[2] Blahut, R. E., 1987. Principle and Practice of Information Theory, reading, MA, Addison-wesley.

[3] Csiszár, I., 1998. Method of types", IEEE Transaction on Information Theory, vol. (44). N 6. pp. 2505-2523.

[4] Csiszár, I. and Körner, J., 1981. Information Theory: Coding Theorem for Discrete Memoryless Systems, Academic press, NewYork.

[5] Csiszár, I. and Shields, P., 2004. Information Theory and Statistics. Fundementals and Trends in Communications and Information Theory, vol. (1), $\mathrm{N}^{\circ} .4$.

[6] Dembo, A. and Zeitouni, O., 1993. Large Deviations Techniques and Applications, Jons and Bartlet. Publishers, London.

Journal home page: www.jafristat.net 
L. Navaei, Journal Afrika Statistika, Vol. 6, 2011, pages 307-315. On error probability exponents of many hypotheses optimal testing illustrations

[7] Haroutunian, E. A., 1988. On asymptotically optimal testing of hypotheses concerning Markov chain", (in Russian). Izvestia Acad. Nauk Armenian SSR. Seria Mathem. vol. 22, $N^{\circ}$. 1. pp. 76-80.

[8] Haroutunian, E. A, Haroutunian, M. E. and Harutyunyan, A. N., 2008. Reliability Criteria in Information Theory and in Statistical Hypothesis Testing", Now Publishers Inc.

[9] Haroutunian E. A. and Navaei, L., 2009. On statistical identification of Markov chain distribution subject to the reliability criterian, Mathematical Problems of Computer Science, vol. 32, pp. 65-69.

[10] Kullback S., 1959. Information Theory and Statistics, Wiley, New York.

[11] Navaei, L., 2007. Large deviations techniques for error exponents to many hypotheses LAO testing, Journal of Modern Applied Statistical Methods, USA, vol. 6, N. 3. pp. 487-491.

[12] Navaei, L., 2010. Identification of Optimality of Multiple Hypotheses Testing, Lambert Academic Publishing, Germany.

[13] Natarajan, S., 1985. Large deviations, hypotheses testing, and source coding for finite Markov chain, IEEE Transaction on Information Theory, vol. 31, $\mathrm{N}^{\circ} .3$, pp. 360-365.

[14] Zeitouni, O. and Gutman, M., 1991. On universal hypotheses testing via large deviations. IEEE Transaction on Information Theory, vol. 37, $\mathrm{N}^{\circ}$. 2, pp.285-290. 